\title{
Cellular senescence in age-related macular degeneration: impact of changes in autophagy and neurotrophic supplementation
}

\author{
Oyuna S. Kozhevnikova \\ Laboratory of molecular mechanisms of \\ aging, Institute of Cytology and Genetics \\ SB RAS, Novosibirsk, Russia \\ oidopova@bionet.nsc.ru
}

\author{
Darya V. Telegina \\ Laboratory of molecular mechanisms of \\ aging, Institute of Cytology and Genetics \\ SB RAS, Novosibirsk, Russia \\ telegina@bionet.nsc.ru \\ Nataliya G Kolosova \\ Laboratory of molecular mechanisms of \\ aging, Institute of Cytology and Genetics \\ SB RAS \\ Novosibirsk, Russia \\ kolosova@bionet.nsc.ru
}

\author{
Mikhail A Tyumentsev \\ Laboratory of molecular mechanisms of \\ aging, Institute of Cytology and Genetics \\ SB RAS, Novosibirsk, Russia \\ landselur@bionet.nsc.ru
}

\begin{abstract}
The senescence-accelerated OXYS rats spontaneously reproduce the major signs of age-related macular degeneration (AMD): dystrophic alterations of the RPE, thinning of the neuroretina, and impairment of choroidal microcirculation. We found increased NGF staining in Muller cells in OXYS rats with progressive stage of retinopathy and differences in the cell typespecific localization of mBDNF between OXYS and Wistar rats. During the development of AMD-like retinopathy, proBDNF dominated over mBDNF. It was suggested that neurons of OXYS rats have a limited ability to process proBDNF, thereby leading to increased cell loss in retina. We showed that the development of AMD-like retinopathy in OXYS rats is accompanied by retinal transcriptome changes affecting genes involved in autophagy. The impaired reactivity of autophagy was confirmed by a decreased number of autophagosomes under the conditions of blocked autophagosome-lysosomal fusion according to immunohistochemical analysis and transmission electron microscopy. Using OXYS rats as a model of AMD, we demonstrated that decreased capacity for upregulation of autophagic flux in response to metabolic stress accompanies the development of AMD and may reflect an age-related decline in the adaptability of retinal cells. Our results indicate disturbances in the neurotrophic support in the retina of OXYS rats. Maintaining sufficient reactivity of autophagy in the retina and the balance of neurotrophic factors may be considered as a strategy to slow down AMD.
\end{abstract}

Keywords - autophagy, neurotrophins, aging, retina, AMD, OXYS rats

\section{Introduction}

Aging is the major risk factor for age-related diseases, including age-related macular degeneration (AMD). AMD is a progressive retinal disorder causing the severe vision impairment in the elderly but the pathophysiology of this disease is still largely unknown. There is increasing evidence that defective proteostasis due to impaired clearance might be the key process in AMD. Autophagy is a conserved cellular degradation pathway for the breakdown of cytoplasmic components: damaged proteins and organelles. Besides a housekeeping function, autophagy is crucial for response to the stress. Defects in the autophagy are linked to aging and disease pathology. Research on the molecular mechanisms underlying the age-related dysregulation of autophagy at the early stage of AMD and before its development can give clues to the most relevant molecular events triggering the entry into the irreversible stage [1].
Molecular studies on AMD are hampered by the inaccessibility of live retinal tissue from AMD patients, especially in the early stages. There is evidence that a suitable experimental model of AMD is senescence-accelerated OXYS rats, which spontaneously develop a phenotype similar to human age-related disorders including AMD-like retinopathy. Retinopathy that develops in OXYS rats already at a young age corresponds (in terms of clinical manifestations and morphological characteristics) to the dry atrophic form of AMD in humans. Nonetheless, neovascularization develops in some $(\sim 10-20 \%)$ of these rats with age. The clinical signs of AMDlike retinopathy appear by the age of 3 months against the background of a reduction in the transverse area of the RPE, impairment of choroidal microcirculation, and retinal thinning. The progression of these abnormalities in OXYS rats is accompanied by a significant reduction in thickness of the photoreceptor cell layer and a decrease in the number of photoreceptor cell nuclei of the outer nuclear layer. Significant pathological changes in the RPE manifest themselves as excessive accumulation of lipofuscin and amyloid in the RPE regions, disturbances in the morphology of the RPE sheet, including an increase in the proportion of multinucleated cells, hypertrophy, distortion of cell shape, and reactive gliosis.

Here, we analyzed high-throughput RNA sequencing (RNASeq) data to identify the specific molecular processes and pathways that take part in the alterations of autophagy during the development and progression of AMD-like retinopathy in OXYS rats. On the basis of previous results revealing an important role of autophagy, we explored the in vivo effects of autophagy activation and inhibition by fasting and chloroquine (CQ) treatment, respectively, on the expression of autophagy markers in the retina of OXYS and control Wistar rats. Also we assess the age-related changes in expression of mNGF, mBDNF, proBDNF and their receptors in the retina of OXYS and Wistar rats.

\section{Methods}

OXYS and Wistar rats at the age of 4 and 16 months were randomly distributed into treatment and control groups $(n=6$ of each genotype per group). The rats consumed feed ad libitum, fasted for 12,24 , or $48 \mathrm{~h}$, or fasted during four daily intraperitoneal injections of CQ (CQ, $50 \mathrm{mg} / \mathrm{kg}$, Sigma-Aldrich, St. Louis, MO, USA) with the last injection administered $3 \mathrm{~h}$ before euthanasia [1]. 
To identify the pathways and biological functions involved in the alteration of autophagy in OXYS rats, we analyzed RNASeq data obtained previously. To identify the GO terms and pathways associated with autophagy over-represented in a DEG list, the detected DEGs were subjected to functional enrichment analyses by means of the DAVID tool with a Benjamini p-value cutoff at $<0.05$. The gene interaction networks related to autophagy were identified on the GeneMANIA web server (http://www.genemania.org/) with default parameters.

Standard techniques of qPCR, immunohistochemistry and electron microscopic examination were used to analyze autophagy modulation effects on retina. Neurotrophins immunofluorescent staining was performed by a standard method [2].

\section{Results}

Here we showed that the development of AMD-like retinopathy in OXYS rats is accompanied by retinal transcriptome changes affecting genes involved in autophagy. These genes are associated with kinase activity, immune processes, and FoxO, mTOR, PI3K-AKT, MAPK, AMPK, and neurotrophin pathways at preclinical and manifestation stages, as well as vesicle transport and processes in lysosomes at the progression stage. Dysregulation of the autophagy transcriptome network in retinal cells takes place already at early stages of AMD-like retinopathy. We demonstrated a reduced response to autophagy modulation (inhibition or induction) in the retina of old OXYS rats: expression of genes Atg5, Atg7, Becn1, Nbrl, Map1lc3b, p62, and Gabarapll differed between OXYS and Wistar (control) rats. The impaired reactivity of autophagy was confirmed by a decreased number of autophagosomes under the conditions of blocked autophagosome-lysosomal fusion according to immunohistochemical analysis and transmission electron microscopy.

We found increased NGF staining in Muller cells in OXYS rats with progressive stage of retinopathy. In contrast, we observed only subtle changes in the labeling of mature BDNF (mBDNF) and TrkB during the development of AMD-like retinopathy in OXYS rats. Using colocalization with vimentin and NeuN, we detected a difference in the cell type-specific localization of mBDNF between OXYS and Wistar rats. We showed that the mBDNF protein was located in Muller cells in OXYS rats, whereas in the Wistar retina, mBDNF immunoreactivity was detected in Muller cells and ganglion cells. During the development of AMD-like retinopathy, proBDNF dominated over mBDNF. It was suggested that neurons of OXYS rats have a limited ability to process proBDNF, thereby leading to increased cell loss in retina..
Currently there is no consensus on whether autophagic activity increases or decreases with age and disease. Apparently, this phenomenon depends on such factors as cell type, disease, or a specific stage of the disease. We have reported that the basal level of autophagy is elevated at the early stage of retinopathy and declines at progressive stages. We demonstrated that retinal autophagy flux is lower in OXYS rats, thereby reducing the capacity of retinal cells to cope with the elevated proteolytic stress in progressive stages of disease. As one of the reasons for the decreased reactivity of autophagy, we propose the accumulation of senescent RPE cells in the retina of OXYS rats. These cells lead to cellular dysfunction and promote the senescence of neighboring cells by secreting the senescenceassociated secretory phenotype. The converse is also possible: autophagy deregulation contributes to senescent cells accumulation. We have observed altered RPE cell morphology in OXYS rats: enlargement, flattening, a loss of the hexagonal shape, accumulation of lipofuscin granules, and multinucleation, which are employed as morphological markers of senescent cells [3].

\section{Conclusion}

Thus, using OXYS rats as a model of AMD, we demonstrated that decreased capacity for upregulation of autophagic flux in response to metabolic stress in the retina accompanies the development of AMD and may reflect an agerelated decline in the adaptability of retinal cells. Alterations of neurotrophin signaling pathway were found at the advanced stage of AMD-like retinopathy development in OXYS rats. Consequently, maintaining sufficient reactivity of autophagy in the retina and the balance of neurotrophic factors may be considered as a strategy to slow down AMD.

\section{ACKNOWLEDGMENT}

This study was supported by the Russian Science Foundation (18-75-00031) and by the Russian Foundation for Basic Research (18-015-00336).

\section{REFERENCES}

[1] Kozhevnikova, O.S.; Telegina, D.V.; Tyumentsev, M.A.; Kolosova, N.G. Disruptions of Autophagy in the Rat Retina with Age During the Development of Age-Related-Macular-Degeneration-like Retinopathy. Int. J. Mol. Sci. 2019, 20, 4804. https://doi.org/10.3390/ijms20194804

[2] Telegina, D.V., Kolosova, N.G. \& Kozhevnikova, O.S. Immunohistochemical localization of NGF, BDNF, and their receptors in a normal and AMD-like rat retina. BMC Med Genomics 12, 48 (2019). https://doi.org/10.1186/s12920-019-0493-8

[3] Telegina, D., Kozhevnikova, O., Bayborodin, S. et al. Contributions of age-related alterations of the retinal pigment epithelium and of glia to the AMD-like pathology in OXYS rats. Sci Rep 7, 41533 (2017). https://doi.org/10.1038/srep41533 